\title{
Experiences of health professionals with nutritional support of critically ill patients in tertiary hospitals in Malawi
}

\section{A Bunyani' ${ }^{1}$ B Mtimuni' ${ }^{2}$ A Kalimbira ${ }^{2}$, P Kamalo $^{3}$}

1. Department of Human Nutrition and Health, Faculty of Food and Human Sciences, Lilongwe University of Agriculture and Natural Resources (LUANAR) and Department of Paediatrics, Kamuzu Central Hospital, Lilongwe, Malawi

2. Department of Human Nutrition and Health, Faculty of Food and Human Sciences, Lilongwe University of Agriculture and Natural Resources (LUANAR), Lilongwe, Malawi

3. Department of Surgery, Faculty of Medicine, College of Medicine, University of Malawi, Blantyre, Malawi

Correspondence to: Arthur Bunyani, Email: bunyaniarthur@gmail.com

\section{Abstract}

\begin{abstract}
Background
Nutritional support is a recognized determinant of outcome in critically ill patients. Development of critical care services in low-income countries has not been accompanied by certain appropriate ancillary services and interventions, such as adequate nutritional support. This study was designed to investigate the experiences of health professionals who have provided nutritional supportive care to critically ill patients admitted to two major central hospitals in Malawi, with the aim of identifying the common practices in nutritional support in these settings.
\end{abstract}

\section{Materials and Methods}

A cross-sectional study in which 50 health professionals working in intensive care and high dependency units, admitting both adult and pediatric patients, were interviewed using a semi-structured questionnaire. Data were coded and then analyzed using SPSS version 16.0. Responses between the two hospitals were compared using Fisher's exact test.

\section{Results}

There was no difference in the composition of respondents from the two hospitals. About $60 \%$ of respondents had had experience with nutritional supplementation in their patients-mainly enteral. The most commonly used formulations were the "ready-to-use therapeutic feeds," followed by modified milk. A high percentage of respondents $(40 \%)$ reported having used dextrose solution as the sole nutritional supplement. Lack of in-service training, nonexistent nutrition protocols pertaining to acutely and critically ill patients, and a lack of clinical nutritionists were the major challenges identified.

\section{Conclusion}

Knowledge of nutrient supplementation was poor among the respondents. The use of ready-to-use therapeutic feeds was quite common, although there is no evidence of its effectiveness in care of acutely critically ill patients. There is a need to establish nutritional support teams in these tertiary hospitals. Clinical nutritionists would ideally help train and play leadership roles in such teams, who would be responsible for assessing patients for their nutritional needs, and ensuring that the feeds provided to patients are appropriate and adequate for their needs.

\section{Introduction}

Adequate nutrition in critically ill patients has been shown to improve patient outcomes, especially those with extreme body mass indices ${ }^{1}$. Several researchers have demonstrated that nutritional support in this patient population reduces critical-care associated infections, duration of mechanical ventilation, and mortality ${ }^{1-3}$. Malnutrition in critically ill patients may be pre-existing or may result from the pathophysiological effects of critical illness, which include the acute inflammatory response resulting in increased nutritional demands; starvation of patients which may be due to physical inability to take food or a requirement after some hospital procedures such as major surgeries; and malabsorption ${ }^{4}$. The majority of hospitalized patients who are also malnourished at the time of admission are unrecognized by hospital personnel and leave hospitals having not received any nutritional therapy ${ }^{4}$. The problem of malnutrition in admitted patients is more serious in developing countries. Waitzberg et al. reported that about half of patients in hospitals in Brazil were malnourished and that physician awareness of malnutrition was low, resulting in under-prescription of nutritional therapy ${ }^{5}$.

Enteral nutrition has emerged as the preferred method of nutritional support in critically ill patients as long as the intestines maintain their digestive and absorptive capacity ${ }^{6}$. If the gut cannot be used for some reason, nutritional therapy may be delivered directly into the venous blood (parenteral nutrition $)^{6,7}$. Whereas parenteral nutrition plays a major role in patients with gastrointestinal failure, enteral nutrition is associated with fewer and less serious complications and is indicated when the gastrointestinal tract is functional. The associated costs of acquiring and administering parenteral nutrition make it unaffordable for most low-income countries who otherwise offer critical care services ${ }^{8}$.

Few empirical data exist on the status and management of critically ill patients in low-income countries like Malawi, and these indicate that very few critically ill patients in these countries have access to appropriate critical care ${ }^{9-14}$. Where available, critical care services are characterized by low staffing levels, lack of appropriate equipment and drugs, high levels of unplanned postoperative surgical admissions, and a high mortality rate, among other challenges ${ }^{9,12,14}$. Little is known about specific nutrition-related interventions and practices geared toward critically ill patients in these settings. At Blantyre's Queen Elizabeth Central Hospital, Malawi's biggest referral and teaching hospital, there are only four intensive care beds representing $0.4 \%$ of all hospital beds? Most critically ill patients who would be treated in the intensive care unit (ICU) if space was available are instead managed in the general wards, in sections dedicated as high dependency units (HDUs), where there is little to no input from critical care staff. The minimal supportive equipment that is available in the HDUs is often non-functional.

This study investigated the experiences of health care professionals providing nutritional support to critically ill patients admitted to both the ICUs and HDUs of two major tertiary hospitals in Malawi, with the aim of identifying the common practices, and potential areas for improvement, related to nutritional support in these settings.

\section{Materials and Methods}

The two hospitals that participated in this study were Kamuzu Central Hospital (KCH) in the Malawi's capital city, Lilongwe, and Queen Elizabeth Central Hospital (QECH) in Blantyre. This was a cross-sectional quantitative study in which a semistructured questionnaire was administered to health personnel working with critically ill paediatric and adult patients in the 
ICUs and HDUs of both aforementioned hospitals. We had calculated that a sample size of 56 participants was required, with a provision for $5 \%$ non-responders. Systematic random sampling was used whereby names of staff working in these units were listed alphabetically and every third person was chosen to participate in the study. Those who were not available were replaced by the next individual. A spectrum of cadres of health professionals was included: medical doctors, nurses, clinical officers, pharmacy technicians, and anaesthetic staff. After coding the data numerically, they were entered in a computer using SPSS16.0 and analyzed. Data analysis included comparisons of responses of participants from the two hospitals. Fisher's exact test at the $95 \%$ confidence level was used to test for significance in any differences in the findings between the staff from the two hospitals. Ethics approval was sought from the National Health Sciences Research and Ethics Committee in Malawi, and the study followed principles of the Declaration of Helsinki 2008. All participants gave written informed consent.

\section{Results}

A total of 50 hospital personnel were interviewed after giving written informed consent: 22 from $\mathrm{KCH}$ and 28 from QECH (Table 1). All of the respondents were experienced staff who had worked in critical care for at least five years. There was no significant difference in the composition of respondents from the two hospitals, with a p-value of 0.1 using Fisher's exact test (Table 1).

Participants were asked about their experiences with nutritional supplementation in acutely critically ill patients in ICU and HDU within the six months preceding the study. About a third reported having used ready-to-use therapeutic feeds (meant for use in nutritional rehabilitation units); some had used milk, which at times was modified by blending with eggs or, very rarely, F75 and F100 milk were used; and others reported some rare special formulations which were salt-free or fat-free preparations from the kitchen (Table 2). Total parenteral nutrition had been used by only three participants, whilst the majority of participants reported only use of dextrose solutions as a nutritional supplementation.

Table 1: Distribution of study participants between the two tertiary hospitals

\begin{tabular}{|l|l|l|l|l|}
\hline $\begin{array}{l}\text { Group of } \\
\text { respondents }\end{array}$ & $\begin{array}{l}\text { Total } \\
\mathbf{N}\end{array}$ & $\begin{array}{l}\text { KCH } \\
\mathbf{n}\end{array}$ & $\begin{array}{l}\text { QECH } \\
\mathbf{n}\end{array}$ & p-value* \\
\hline Nurses & 15 & 9 & 6 & 0.151 \\
\hline Medical doctors & 13 & 4 & 9 & \\
\hline Clinical officers & 12 & 5 & 7 & \\
\hline Anaesthetic staff & 4 & 3 & 1 & \\
\hline $\begin{array}{l}\text { Pharmacy } \\
\text { Technicians }\end{array}$ & 6 & 1 & 5 & \\
\hline TOTALS & 50 & 22 & 28 & \\
\hline
\end{tabular}

* Fisher's exact test used for $p$-values

The participants identified four major challenges affecting their ability to provide nutrition support effectively: (1) lack of a nutrition department with qualified nutritionists in their respective hospitals; (2) lack of capacity in hospital pharmacies to prepare or procure standard nutritional supplements for patients; (3) lack of comprehensive training in nutrition whilst in college and at work; and (4) absence of nutritional protocols for acutely critically ill adult patients in the wards.

Participants were further asked about their perceptions of the (potential) role of a clinical nutritionist in the patient management team. They responded that clinical nutritionists in the hospitals could be involved in assessing the nutritional status of patients based on anthropometric measurements, making requests for feeds from the pharmacy, and conducting nutrition training. Some participants also mentioned that they felt clinical nutritionists could act as coordinators of nutrition programs at the hospitals and at a national level.

Table 2: Participants experience with use of nutrition supplementation over a period of 6 months

\begin{tabular}{|l|l|l|l|l|}
\hline $\begin{array}{l}\text { Nutrition } \\
\text { supplement } \\
\text { experience }\end{array}$ & $\begin{array}{l}\text { Total } \\
\mathbf{N}\end{array}$ & $\begin{array}{l}\text { KCH } \\
\mathbf{n}\end{array}$ & $\begin{array}{l}\text { QECH } \\
\mathbf{n}\end{array}$ & p-value* \\
\hline Enteral feeds & & & & \\
\hline $\begin{array}{l}\text { Ready-to-use } \\
\text { therapeutic } \\
\text { feeds (RUTF) }\end{array}$ & 17 & 9 & 8 & 0.104 \\
\hline Milk $^{* *}$ & 6 & 2 & 4 & \\
\hline $\begin{array}{l}\text { Rare special } \\
\text { formulations }\end{array}$ & 4 & 4 & 0 & \\
\hline $\begin{array}{l}\text { Total } \\
\text { parenteral } \\
\text { nutrition }\end{array}$ & 3 & 3 & 0 & \\
\hline $\begin{array}{l}\text { Dextrose } \\
\text { infusion only }\end{array}$ & 20 & 6 & 14 & \\
\hline Total & 50 & 24 & 26 & \\
\hline
\end{tabular}

* Fisher's exact test used for p-values

** Includes plain milk with or without blended egg, and therapeutic milk (F75 and F100),

***Salt-free and fat-free preparations from the hospital kitchen

\section{Discussion}

In this study it was found that, in the two tertiary hospitals, the mainstay of nutritional support was enteral feeding using RUTF. At QECH, some patients were receiving milk which, depending on availability, was modified by blending with eggs; but respondents from Lilongwe did not mention such a preparation. Exclusive to $\mathrm{KCH}$ was the use of other special enteral formulations. In addition, few participants from $\mathrm{KCH}$ reported the rare access to parenteral nutrition including amino acid infusions, which was not reported at QECH. Although some patients with gut failure may benefit from parenteral nutrition, the predominance of enteral nutrition observed in this study is desirable and in line with international trends ${ }^{6}$. Parenteral nutrition is not locally available in Malawi, and the few participants who had had some experience with it used donated supplies; we therefore will not further discuss parenteral nutrition.

What needs to be addressed is whether the preparations given to patients in these hospitals (RUTF and milk) are suitable for critically ill patients and sufficient to avoid protein-energy undernutrition in this patient population. RUTF is an innovative food product consisting of peanuts, and is enriched with sugar, powdered milk, vegetable oil, vitamins, and minerals and is considered a high-energy dense food and currently forms the standard of care in nutrition rehabilitation of severely and moderately malnourished children ${ }^{15-17}$. The product has also been used in malnourished 
adult patients with human immunodeficiency virus (HIV) starting antiretroviral treatment in Malawi with considerable success in improving patient weight, though not reducing mortality in the HIV patients initiating antiretroviral therapy ${ }^{18,19}$. RUTF has also been used in pregnant women ${ }^{20}$.

The challenge with the use of RUTF in acutely critically ill patients is that the product was originally designed for severe malnutrition in young children with the accompanying pathophysiological changes in mind; which are quite different from the changes in acutely critically ill patients. With some exceptions, like in patients with renal, hepatic, or respiratory failure, it is recommended that acutely critically ill patients receive a moderate amount of energy comprising 30 to $70 \%$ carbohydrate source, 20 to $50 \%$ lipid source and 15 to $20 \%$ protein source ${ }^{20,21}$. The recommended daily protein intake in acutely critically ill patients is 1.2 to $1.5 \mathrm{~g} / \mathrm{kg}$ body weight ${ }^{20,21}$. Contrary to these guidelines, RUTF's energy composition is $32 \%$ from its carbohydrate component, 58\% from its lipid component, and only $10 \%$ from its protein component ${ }^{15}$. Thus, for a critically ill patient on exclusively RUTF nutrition supplementation, the amount of RUTF that would provide the daily required energy would only provide $0.77 \mathrm{~g}$ of protein per $\mathrm{kg}$ body weight, which is only half the required amount of protein in this condition. In addition, guidelines for preparation of nutrition formulas of critically ill patients recommend the use of small amounts of micronutrients ${ }^{20,21}$ RUTFs, however, are loaded with micronutrients-in most cases over 100 times the daily recommended intake ${ }^{15}$. This makes RUTF less appropriate as a nutrition source for critically ill patients. There is a need to explore other nutritional options that are more appropriate for the needs of critically ill patients.

Another point of concern in the findings was the supposition by many of the critical care staff that intravenous dextrose solutions are a form of nutritional supplementation. This finding was more prevalent at QECH where over half the respondents mentioned only having had experience with dextrose as a form of nutritional support (Table 2). Although dextrose solution provides energy, it does not contain any other nutrients necessary for the critically ill patient, and assuming such is the case could be a result of inadequate training and lack of in-service training, which was also noted by the respondents. Lack of in-service training was also cited by Mula as a major contributor to the variability in levels of knowledge among nurses working in the intensive care and high dependency units at one major hospital in Malawi ${ }^{22}$. Provision of inappropriate nutrition can also be compounded by the lack of nutritional protocols for acutely critically ill patients and the absence of nutrition support teams for such patients in the two hospitals studied. Nutritional protocols or algorithms have been shown to improve the quality of nutritional support in critically ill patients $^{23}$. The amount of calories prescribed to patients, and those subsequently delivered, increased significantly by almost 20\% after instituting a nutritional algorithm in one intensive care unit ${ }^{23}$. Fulbrook et al., also concluded that in hospitals with nutritional support teams, more thorough nutritional risk assessments were done ${ }^{24}$. Development of nutritional protocols and support teams critically ill patients can therefore improve the level of knowledge in nutrition and hence improve the care given to patients in these two hospitals. The respondents in our study showed appreciation of the role of nutritionists in the patient care team and such positive attitudes could be grounds for collaboration between nutritionists and the mainstream patient management teams.

\section{Study Limitations}

Although this study gives a good overview of nutritional support given to critically ill patients in Malawi, the findings are more inductive since we did not primarily find out what the patients were actually receiving in the units. Secondly, the respondents may have known of other forms of nutrition but we only asked them what they had commonly used in the six months under review. The requirement to recall what the critical care staff had used in the six months preceding the study may have also introduced a recall bias. Despite these limitations, the study has managed to extract the general knowledge of the participants on nutritional support, which most likely informs their day-to-day practice.

\section{Conclusions}

A limited number of nutritional supplementation options are in use for acutely critically ill patients at the two tertiary hospitals assessed. There is no systematic use of these preparations, and there is need to find out if the options available are appropriate for the needs of this particular group of patients. The study has also revealed several challenges facing health professionals in the provision of nutritional support including lack of proper adult and paediatric nutrition departments in the hospitals, lack of trainingboth long and short term, lack of resources to help in the management of cases, lack of nutritional protocols to guide when to stop or introduce another nutritional intervention, as well as lack of nutritional education to both patients and health professions. Most respondents indicated that the presence of clinical nutritionists would go a long way in mitigating the challenges in nutrition currently faced by these hospitals.

\section{Recommendations}

The following recommendations are proposed as a way forward in advancing the nutrition of critically ill patients in Malawi.

There is need for a well-designed comprehensive prospective study to assess the effectiveness and impact of the current nutritional interventions. Such a study may include assessment of the patients' nutritional requirements, and correlation of these requirements with what patients actually receive and with patient outcomes. There is also need to develop lowcost nutritional supplements from locally available food sources, which can be used for critically ill patients.

Central hospitals should incorporate clinical nutrition specialists in the care of hospitalized patients, including critically ill patients, in addition to the therapeutic services for childhood malnutrition currently offered by these central hospitals. Engaging clinical nutritionists would ensure appropriate and timely nutritional interventions in critically ill patients; increased awareness of, and ongoing training in, nutrition requirements; and management of critically ill patients, by health care professionals looking after these patients.

\section{References}

1. Alberda C, Gramlich L, Jones N, et al. The relationship between nutritional intake and clinical outcomes in critically ill patients: results of an international multicenter observational study. Intensive Care Medicine. 2009;35:1728-1737 DOI:10.1007/s00134-009-1567-4. doi:10.1007/s00134-009-1567-4. 
2. Artinian V, Krayem H, DiGiovine B. Effects of Early Enteral Feeding on the Outcome of Critically Ill Mechanically Ventilated Medical Patients. Chest. 2005;129:960-967.

3. Marik PE, Zaloga GP. Early enteral nutrition in acutely ill patients: A systematic review. Critical Care Medicine. 2001;29(12).

4. Prins A, Sa MRD. Nutritional assessment of the critically ill patient. South African Journal of Clinical Nutrition. 2010;23(1):11-18.

5. Waitzberg DL, Caiaffa WT, Correia MITD. Hospital Malnutrition : The Brazilian National Survey ( IBRANUTRI ): A Study of 4000 Patients. Nutrition. 2001;17:573-580.

6. Jeejeebhoy KN. Parenteral nutrition in the intensive care unit. Nutrition reviews. 2012;70(11):623-30. doi:10.1111/j.1753-4887.2012.00538.x.

7. Fletcher J. Parenteral nutrition: indications, risks and nursing care. Nursing Standard. 2013;27(46):50-58.

8. Poole R, Gillanders L, Plank L, Mcilroy K, Davoren H, Parry B. Economic analysis model for inpatient parenteral nutrition: A pilot study. Nutrition \& Dietetics. 2009;66(4):221-226. doi:10.1111/j.17470080.2009.01374.x

9. Size M, Borgstein E, Haisma H. One-year audit of admissions to the Intensive Care Unit of Queen Elizabeth Central Hospital, Blantyre. Malawi Medical Journal. 2005;17(1):12-14.

10. Ilori IU, Kalu QN. Intensive care admissions and outcome at the University of Calabar Teaching Hospital, Nigeria. Journal of Critical Care. 27AD;1:105-e1 DOI: 10.1016/j.jcrc.2011.11.011.

11. Jochberger S, Ismailova F, Lederer W, et al. Anesthesia and its allied disciplines in the developing world: a nationwide survey of the Republic of Zambia. Anesthesia and Analgesia. 2008;106(3):942-8. doi:10.1213/ane.0b013e318166ecb8.

12. Dünser MW, Baelani I, Ganbold L. A review and analysis of intensive care medicine in the least developed countries. Critical Care Medicine 2006;34(4):1234-1242. doi:10.1097/01.CCM.0000208360.70835.87.

13. Dünser MW. Intensive care medicine in resource-limited settings: a general overview. Updates in Anaesthesia. 2012;28:7-10.

14. Towey RM, Anyai JB. Intensive care medicine in rural sub-Saharan Africa - who to admit? Updates in Anaesthesia. 2012;28:18-21.
15. Santini A, Novellino E, Armini V, Ritieni A. State of the art of Ready-to-Use Therapeutic Food: a tool for nutraceuticals addition to foodstuff. Food Chemistry. 2012:doi: http://dx.doi.org/ 10.1016/j. foodchem.2012.10. doi:10.1016/j.foodchem.2012.10.098.

16. Andre B. Highly nutrient-dense spreads: a new approach to delivering multiple micronutrients to high-risk groups. British Journal of Nutrition. 2001;85(Suppl. 2):S175-S179. doi:10.1049/BJN2000311.

17. Children W, Matilsky DK, Maleta K, Castleman T, Manary MJ. Supplementary Feeding with Fortified Spreads Results in Higher Recovery Rates Than with a Corn / Soy Blend in Moderately. Journal of Nutrition. 2009;139:773-778. doi:10.3945/jn.108.104018.severe.

18. Ndekha M, van Oosterhout JJ, Zilstra EE, Manary M, Saloojee H, Manary MJ. Supplementary feeding with either ready-to-use fortified spread or corn-soy blend in wasted adults starting antiretroviral therapy in Malawi: randomised, investigator blinded, controlled trial. British Medical Journal. 2009;338(b1867):doi:10.1136/bmj.b1867. doi:10.1136/bmj.b1867.

19. Manary M, Ndekha M, van Oosterhout JJ. Supplementary feeding in the care of the wasted HIV infected patient. Malawi Medical Journal. 2010;22(June):46-48. doi:10.1086/605285.5.

20. Pichard C, Biolo G, Planas M, Roth E, Schols A. Enteral nutrition in intensive care patients : a practical approach. Intensive Care Medicine. 1998;24:848-859.

21. Webster NR, Galley HF. Nutrition in the critically ill patient. Journal of the Royal College of Surgeons of Edinburg. 2000;45(December):373-379.

22. Mula C. Nurses' Competency and Challenges in Enteral feeding in the Intensive Care Unit (ICU) and High Dependency Units (HDU) of a referral hospital, Malawi. Malawi Medical Journal. 2014;26(3):Published online on 06.09.2014.

23. Wøien H, Bjørk IT. Nutrition of the critically ill patient and effects of implementing a nutritional support algorithm in ICU. Journal of Clinical Nursing. 2006;15:168-177.

24. Fulbrook P, Bongers A, Albarran JW. A European survey of enteral nutrition practices and procedures in adult intensive care units. Journal of Clinical Nursing. 2007;16:2132-2141. doi: 10.1111/j.1365-. doi:10.1111/j.1365-2702.2006.01841.x. 